\title{
ANEMIA EN FUTURAS GENERACIONES MÉDICAS
}

\author{
ANEMIA IN FUTURE MEDICAL GENERATIONS
}

Leyla Rodas-Alvarado 1,a

\section{Sr. Editor}

La anemia es un trastorno en el cual el número de eritrocitos es insuficiente para satisfacer las necesidades del organismo viéndose afectada la capacidad de transporte de oxígeno en la sangre ${ }^{(1)}$. La anemia puede conducir al desarrollo de dolor de cabeza, fatiga, letargo, apatía, disnea de esfuerzo, pal pitaciones y tinnitus y, por lo tanto, disminuir la calidad de la vida cotidiana de sobremanera. Tales síntomas pueden representar un obstáculo para los estudiantes en el trascurso de su vida académica y tener un impacto negativo en su carrera $^{(2)}$.

Este problema se enfrenta con mayor frecuencia en mujeres de 18 a 25 años. Los estudiantes de medicina, especialmente las mujeres que viven dentro de la universidad, presentan un alto riesgo de anemia debido a sus malos hábitos alimenticios, falta de desayuno, horario prolongado en la universidad, carga de estudios médicos, investigación científica, además de actividades extracurriculares ${ }^{(3)}$.

Dado que la anemia está asociada con malos hábitos alimenticios y aunque la anemia es causada principalmente por la deficiencia de hierro, la baja capacidad de transporte de oxígeno puede deberse a otras afecciones como enfermedades crónicas, que siguen siendo un problema de salud relevante en Estados Unidos inclusive(4).

Se realizó un estudio transversal en el Instituto Adichunchanagiri de Ciencias Médicas, donde fueron incluidos doscientos ochenta y nueve estudiantes de medicina de ambos sexos. La mayoría de los participantes pertenecían al grupo de edad de 17 a 20 años (84,4\%). La prevalencia general de anemia fue del $15,6 \%$, donde curiosamente, el nivel medio de hemoglobina fue mayor entre los estudiantes de bajo rendimiento, lo que indica, según el autor, que la anemia nutricional puede no necesariamente desempeñar un papel importante en el rendimiento educativo de nivel superior ${ }^{(2)}$.

Se realizó otro estudio transversal en 200 estudiantes mujeres aparentemente sanas (entre 19 y 25 años) de la Universidad de Tabuk, Arabia Saudita, para investigar la anemia con deficiencia de hierro, así como su prevalencia y factores de riesgo. Se recopilaron datos sobre los índices sociodemográficos, de dieta, de salud, de antropometría y de estado de hierro hematológico y bioquímico de los participantes. La prevalencia de la deficiencia de hierro fue del 12,5\%. Los factores asociados mediante regresión logística con un riesgo elevado de anemia fueron, ingestas inadecuadas de hierro y vitamina $C$, consumo infrecuente de carne roja ( $\leq 2$ veces por semana), consumo frecuente de té ( $\geq 2$ veces por semana) y antecedentes personales de deficiencia de hierro( ${ }^{(5)}$.

Con lo redactado se puede mencionar que podría existir una asociación entre el estilo de vida de los estudiantes de medicina y el déficit de hemoglobina, como también, podría estar relacionado el desempeño académico. Por lo que se plantea como alternativa de solución, capacitación a los alumnos de medicina en temas sobre nutrición y estilos de vida saludable, medidas de prevención que incluirían exámenes de sangre donde se vería la hemoglobina y el hematocrito para un diagnóstico y tratamiento rápido para así evitar que esta patología silente no solo pueda que afecte el desempeño académico propiamente dicho, sino también el desarrollo personal.

' Facultad de Medicina Humana, Universidad Ricardo Palma, Lima - Perú.

a Estudiante de medicina Humana.

Citar como: Leyla Rodas-Alvarado. Anemia en futuras generaciones médicas. Rev. Fac. Med. Hum. Abril 2020; 20(2):337-338. DOI 10.25176/RFMH. V20i2.2281 
Contribuciones de autoría: La autora realizó la Conflicto de interés: La autora declara no tener generación, recolección de información, redacción y versión final del artículo original.

Financiamiento: Autofinanciado. conflictos de interés en la publicación de este artículo.

Recibido: 25 de setiembre 2020

Aprobado: 07 de febrero 2020

Correspondencia: Leyla Rodas-Alvarado.

Dirección: Calle José Olaya 164, José Carlos Mariátegui, Villa María del Triunfo, Lima - Perú.

Teléfono: 51941482307

Correo: leyla.rodas.alvarado@gmail.com

\section{REFERENCIAS BIBLIOGRÁFICAS}

1. Organización Mundial de la Salud. Concentraciones de hemoglobina para diagnosticar la anemia y evaluar su gravedad. Ginebra, Organización Mundial de la Salud, 2011 (WHO/NMH/NHD/MNM/11.1) http://www.who.int/vmnis/ indicators/haemoglob in_es.pdf

2.Rani NA, Arasegowda R, Mukherjee P, Dhananjay SY. Prevalencia de la anemia por deficiencia nutricional y su impacto en el rendimiento escolar entre estudiantes de medicina de pregrado. J Clin Diagnóstico Res. 2017

11 (3): BC21 - BC23. https://www.ncbi.nlm.nih.gov/pubmed/28511373 doi: 10.7860 / JCDR / 2017 / 25367.9597

3. Jawed S, Tariq S, Tariq S, Kamal A. Frecuencia de anemia nutricional entre las estudiantes de medicina de Faisalabad. Pak J Med Sci. 2017; 33 (2): 398-403. https://www.pjms.com.pk/index.php/pjms/article/view/11854 doi: 10.12669 / pjms.332.11854

4. Le $\mathrm{CH}$. The Prevalence of Anemia and Moderate-Severe Anemia in the US Population (NHANES 2003-2012). PLoS One. 2016;11(11): e0166635.

https://journals.plos.org/plosone/article?id=10.1371/journal.pone.0166635. doi:10.1371/journal.pone.0166635

5. Alzaheb RA, Al-Amer O. La prevalencia de la anemia por deficiencia de hierro y sus factores de riesgo asociados entre una muestra de estudiantes universitarias en Tabuk, Arabia Saudita. Clin Med Insights Women's health. 2017; 10: 1179562X17745088. https://journals.sagepub.com/ doi/10.1177/1179562X17745088. doi: 10.1177 / 1179562X17745088

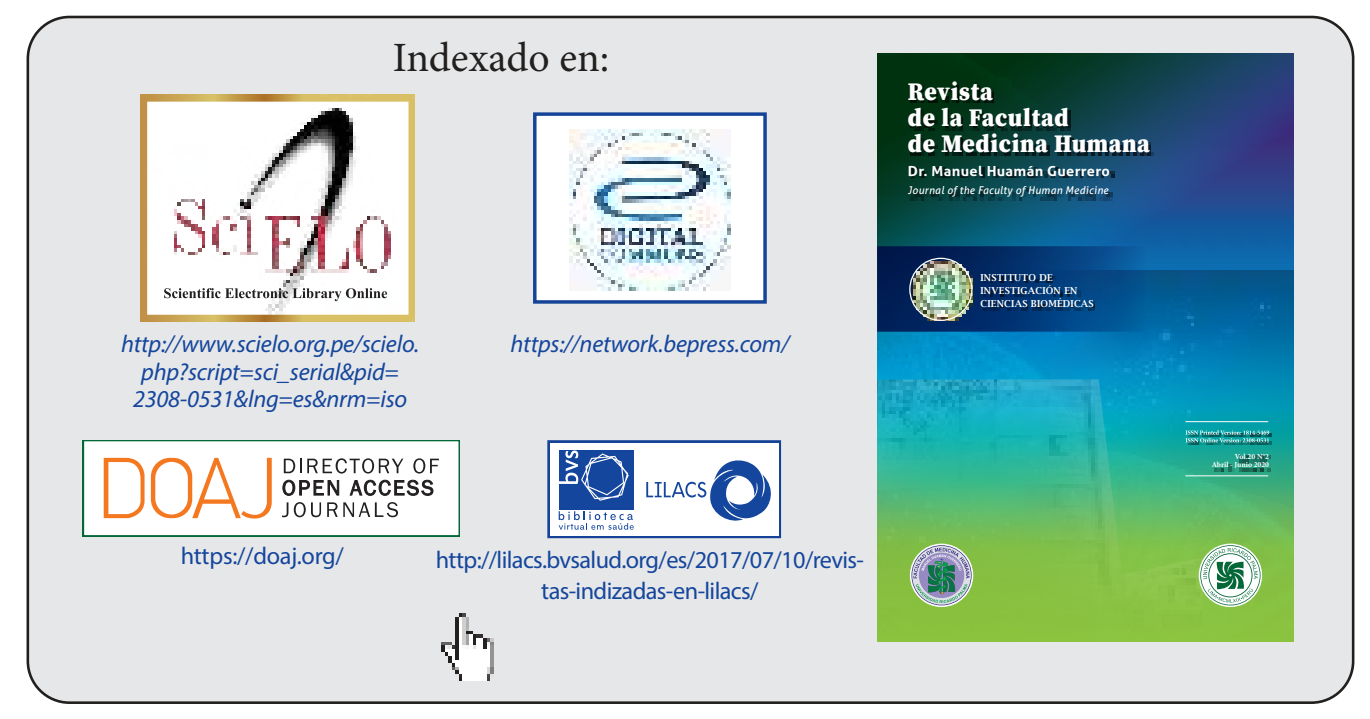

